\title{
Enterocyte zonation along the intestinal villus reveals a division of labour
}

The researchers measured gene expression in five zones from the bottom to the top of the villus

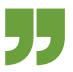

A variety of tasks such as nutrient absorption and protection from pathogenic bacteria are performed at the intestinal villus by enterocytes, but whether these tasks are spatially zonated was unknown. Researchers have now uncovered broad zonation patterns along the villus, showing that enterocytes, previously considered terminally differentiated, undergo transdifferentiation as they migrate across the villus axis.

In the gut, it is well-known that stem cells in intestinal crypts generate enterocytes that migrate along the villus until they are shed at the villus tip. "Once cells have exited the crypt, we usually consider them as terminally differentiated cells. We questioned whether this was really the case," explains author Shalev Itzkovitz. "The villus is a graded microenvironment, with key factors varying from the crypt to the villus tip. It could be that not all enterocytes perform all functions, but rather different zones along the villus specialize in distinct functions."

The Itzkovitz lab specializes in using laser capture microdissection

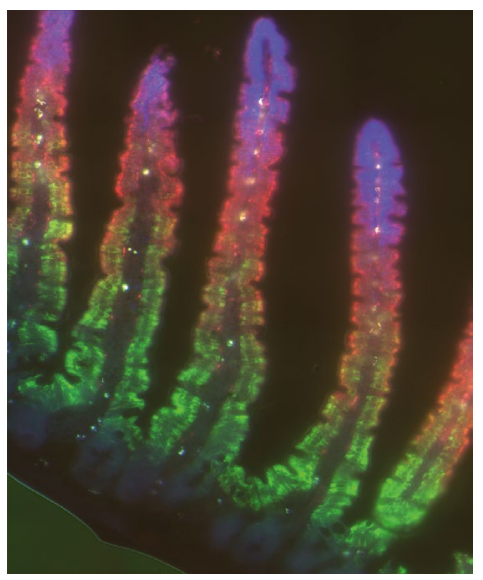

Fluorescent in situ hybridisation staining of villus enterocytes: REG1 in green, SLC2A2 in red and ADA in blue. Reproduced with permission from Moor, A.E. et al. (2018), Elsevier.
(LCM) techniques with highcoverage transcriptional assays to measure gene expression in welldefined zones from very small tissue areas. By applying this approach to the intestinal villus and measuring expression profiles of zones along the villus axis, they could address the question of whether enterocyte gene expression is zonated.

The researchers measured gene expression in five zones from the bottom to the top of the villus in samples of mouse jejunum. "We were surprised to find substantial gradients of gene expression, with many genes increasing and decreasing in expression from villus base to villus tip," reports Itzkovitz. However, this approach had limitations, such as mRNA degradation from tissue handling and impurities from the presence of multiple cell types. "To overcome these challenges, we combined LCM with single-cell RNA sequencing, which provides the transcriptomes of thousands of single cells," says Itzkovitz. "It is then straightforward to filter out non-enterocytes computationally by clustering according to gene expression."

Now with pure enterocyte data, the team devised an algorithm to examine the expression of a panel of landmark genes that were identified from the earlier LCM experiments to precisely localize each sequenced enterocyte to a coordinate position on the villus axis. Obtaining data from hundreds of enterocytes from each villus coordinate enabled the generation of a genome-wide map of enterocyte gene expression.

"We were surprised that $>80 \%$ of enterocyte genes were zonated," says Itzkovitz. The investigators found that enterocytes at the bottom of the villus express antimicrobial sensing machinery and secreted antimicrobial peptides in a microbiome-dependent manner. "Assigning this function to the bottom of the villus makes sense, as these antimicrobial peptides might reduce the entry of bacteria to the sensitive crypt compartment. These villus-bottom enterocytes serve as gatekeepers to prevent damage to the crypts," explains Itzkovitz.

Moving up the villus, they found enterocytes shut down the antimicrobial programmes and upregulate nutrient transporters, finding the machinery to absorb different nutrients expressed in spatially distinct compartments. Amino acid transporters were expressed at the bottom of the villus before a shift to carbohydrate transporters and then apolipoproteins, necessary for lipid absorption, were expressed at the villus tops.

"What are the mechanisms that give rise to these dramatic zones of gene expression," asks Itzkovitz. "We believe the answer resides in the mesenchymal cells within the villus and we are actively working to define the molecular signatures of these cells at different villus zones." The researchers are also interested in whether the zonation of nutrient transporters generates zonated bacterial communities and whether expression profiles change in response to diet and pathologies such as IBD. "Finally, we are working to go beyond mRNA with the aim of characterizing the complete epigenome, proteome and metabolome of enterocytes along the villus axis," concludes Itzkovitz.

Iain Dickson 